UPR-1029-T, PUPT-2078, hep-th/0303197

\title{
More Supersymmetric Standard-like Models from Intersecting D6-branes on Type IIA Orientifolds
}

\author{
Mirjam Cvetič ${ }^{1}$ and Ioannis Papadimitriou ${ }^{2}$ \\ ${ }^{1}$ School of Natural Sciences, Institute for Advanced Studies, Princeton NJ 08540 USA and \\ Department of Physics and Astronomy, \\ Rutgers University, Piscataway, NJ 08855-0849 USA* \\ ${ }^{2}$ Princeton University, Princeton NJ $08540 U_{S A}^{\dagger}$
}

(Dated: November 19, 2018)

\begin{abstract}
We present new classes of supersymmetric Standard-like models from type IIA $\mathbf{T}^{\mathbf{6}} /\left(\mathbf{Z}_{\mathbf{2}} \times \mathbf{Z}_{\mathbf{2}}\right)$ orientifold with intersecting D6-branes. D6-branes can wrap general supersymmetric three-cycles of $\mathbf{T}^{\mathbf{6}}=\mathbf{T}^{2} \times \mathbf{T}^{\mathbf{2}} \times \mathbf{T}^{\mathbf{2}}$, and any $\mathbf{T}^{\mathbf{2}}$ is allowed to be tilted. The models still suffer from additional exotics, however we obtained solutions with fewer Higgs doublets, as well as models with all three families of left-handed quarks and leptons arising from the same intersecting sector, and examples of a genuine left-right symmetric model with three copies of left-handed and right-handed families of quarks and leptons.
\end{abstract}

${ }^{*}$ On Sabbatic Leave from the University of Pennsylvania

${ }^{\dagger}$ Exchange Scholar from the University of Pennsylvania 


\section{INTRODUCTION}

The intersecting D-brane configurations of Type II string theory, compactified on orientifolds play an important role in the construction of four-dimensional solutions $[1,2,3,4,5]$. In particular the appearance of the chiral matter $[6,7]$ at the brane intersection provides a promising starting point to construct the models with potentially quasi-realistic particle physics features.

Techniques to construction of intersecting D-brane models were developed, primarily for non-supersymmetric constructions, in $[1,2,3,4,5]$ (and subsequently explored in $[8,9,10$, 11]). Many non-supersymmetric models with quasi-realistic features of the Standard-like and grand-unified models can be obtained, satisfying the Ramond-Ramond tadpole cancellation conditions. However, since the models are non-supersymmetric, there are uncancelled NeveuSchwarz-Neveu-Schwarz tadpoles as well as the radiative corrections at the effective theory level, which are of the string scale. Since the intersecting D6-branes typically have no common transverse in the internal space, the string scale is of the Planck scale. Therefore the tree-level constructions typically suffer from large Planck scale corrections at the loop level.

On the other hand, constructions of supersymmetric models turn out to be extremely constraining. The first supersymmetric model with intersecting D6-branes and the features of the supersymmetric Standard-like models has been constructed [12, 13, 14]. They are based on the $\mathbf{Z}_{\mathbf{2}} \times \mathbf{Z}_{\mathbf{2}}$ orientifold with D6-branes wrapping specific supersymmetric threecycles of the six-torus. Interestingly, the embedding of supersymmetric four-dimensional models with intersecting D6-branes has a lift $[13,14]$ into M-theory that corresponds to the compactification of M-theory on a singular $G_{2}$ holonomy manifold $[12,13,15,16,17]$.

Within the above class of models examples of supersymmetric four-family [13] and threefamily [19] SU(5) models were also constructed. In particular, in [19] a systematic analysis for three-family models with D6-branes wrapping general three-cycles of internal six-torus and for general tilted two-tori was given. There are of the order of 80 models in this class of constructions and they all necessarily contain three-copies of $\mathbf{1 5}$ of SU(5).

Recently three-family supersymmetric left-right symmetric models based on $\mathbf{Z}_{\mathbf{4}}[20]$ and $\mathbf{Z}_{\mathbf{4}} \times \mathbf{Z}_{\mathbf{2}}$ [21] orientifolds with intersecting D6-branes were obtained. In these models the left-right symmetric gauge structure was obtained via brane recombinations and thus the final model does not have an explicit toroidal orientifold construction, where conformal field theory techniques could be applied for the calculation of the full spectrum and couplings.

While providing an explicit example of Standard-like model gauge group and three copies of quarks and leptons, the original construction $[12,13]$ suffers from a number of phenomenological difficulties. One is the existence of the adjoint matter on each brane, which is a general 
property supersymmetric as well as non-supersymmetric toroidal orientifold constructions. It is due to the fact that the typical cycles of the tori, wrapped by D6-branes, are not rigid. Addressing Calabi-Yau compactifications with rigid supersymmetric cycles would therefore be one avenue to pursue, however the calculational techniques of conformal field theory may not be applicable there. The original construction also contains charged exotics, some of them fractionally charged, and it has many Standard-Model Higgs doublets $\left(12 H_{U}\right.$ and 12 $\left.H_{D}\right)$. The model also has two additional non-anomalous $\mathrm{U}(1)$ factors, and for one of them there are no light elementary Higgs field candidates to spontaneously break it. (See [22] for details.)

The model, however, has an additional quasi-hidden gauge sector that is confining. This sector may allow for binding of exotics into composite states that have charge assignments of the (fourth) left-handed family, as discussed in [22]. Another interesting implication of this confining sector is a possibility of gaugino condensation which may trigger supersymmetry breaking and stabilization of moduli [24].

Phenomenology of both non-supersymmetric $[4,11,25]$ and supersymmetric $[22,23,24]$ models was addressed, however the calculations of couplings has been only recently studied. The tree-level gauge couplings and their low energy implications have been studied (See e.g., [22] and references therein). A calculation of gauge coupling threshold corrections [26] is also of interest, since it could be compared to the strong coupling limit of M-theory compactified on the corresponding $G_{2}$ holonomy space [18].

Most recently, progress has been made for the calculations of couplings for states at the intersections. In particular the Yukawa couplings of quarks and leptons to Higgs fields is of most interest. These couplings are expected to obtain contributions from the world-sheet (disk) instantons associated with the open strings stretching between the three intersections [2]. Recently, the complete instanton sum for toroidal orientifolds has been addressed in [27]. The full coupling, that includes the classical and the quantum part contribution has been calculated in [28], employing the conformal field theory technique. (See also [29] for a conformal field theory calculation of the classical part of the Yukawa couplings.)

The purpose of this paper is to present new classes of supersymmetric Standard-like models within $\mathbf{T}^{\mathbf{6}} /\left(\mathbf{Z}_{\mathbf{2}} \times \mathbf{Z}_{\mathbf{2}}\right)$ orientifolds with intersecting D6-branes. We generalize the original constructions $[12,13]$ by allowing for D6-branes wrapping most general three-cycles, constructed as a product of one-cycles on each two-torus, and for any of the two-tori to be tilted. The aim is to demonstrate existence of Standard-like models with fewer Higgs doublets and fewer exotics. As a by-product we also obtain the models that have threefamilies of left-handed quarks and leptons arising for the same intersecting sector, as well as the models where the number of the right-handed families is also three. [In the original construction one of the families arises from a different intersection sector. There the number 
of right-handed families was even, which in turn required additional exotics to cancel the Standard-Model gauge group anomalies.]

The models that we constructed are basically descendants of the left-right symmetric models, i.e. the non-anomalous hypercharge is obtained as a linear combination of nonanomalous U(1) charges that lie within the Pati-Salam left-right gauge group structure [30]. As a consequence we typically obtain two additional non-anomalous U(1) gauge group factors in the observable sector, just as in the original construction.

The paper is organized as follows. In Section II we summarize the features of the construction. In Section III we give details about specific classes of new models. Conclusions and open avenues are given in Section IV.

\section{CONSTRUCTION OF SUPERSYMMETRIC MODELS FROM A $\mathrm{T}^{6} / \mathrm{Z}_{2} \times \mathrm{Z}_{2}$ ORIENTIFOLD WITH INTERSECTING D6-BRANES}

Let us, for completeness, present the orientifold construction in [13] as well as some minor changes in notation which were introduced in [19]. The starting point is type IIA string theory compactified on a $\mathbf{T}^{\mathbf{6}} /\left(\mathbf{Z}_{\mathbf{2}} \times \mathbf{Z}_{\mathbf{2}}\right)$ orientifold. We take $\mathbf{T}^{\mathbf{6}}=\mathbf{T}^{\mathbf{2}} \times \mathbf{T}^{\mathbf{2}} \times \mathbf{T}^{\mathbf{2}}$ to be the product of three 2 -tori and introduce complex coordinates $z_{i}, i=1,2,3$ parameterizing each of the 2 -tori. The orbifold group generators $\theta, \omega$ then act on $\mathbf{T}^{\mathbf{6}}$ as

$$
\begin{aligned}
& \theta:\left(z_{1}, z_{2}, z_{3}\right) \rightarrow\left(-z_{1},-z_{2}, z_{3}\right) \\
& \omega:\left(z_{1}, z_{2}, z_{3}\right) \rightarrow\left(z_{1},-z_{2},-z_{3}\right) .
\end{aligned}
$$

The orientifold projection is implemented by gauging the symmetry $\Omega R$, where $\Omega$ is worldsheet parity, and $R$ acts as

$$
R:\left(z_{1}, z_{2}, z_{3}\right) \rightarrow\left(\bar{z}_{1}, \bar{z}_{2}, \bar{z}_{3}\right) .
$$

There are then four kinds of orientifold 6-planes (O6-planes), associated with the actions of $\Omega R, \Omega R \theta, \Omega R \omega$, and $\Omega R \theta \omega$ respectively. To cancel the RR charge of the O6-planes, D6-branes wrapped on factorized three-cycles are introduced. We consider $K$ stacks of $N_{a}$ D6-branes, $a=1, \ldots, K$, wrapped on the $n_{a}^{i}\left[a_{i}\right]+m_{a}^{i}\left[b_{i}\right]$ cycle in the $i^{t h}$ two-torus. There are only two choices of the complex structure consistent with the orientifold projection as explained in [19]. In either case a generic two cycle is labelled by $\left(n_{a}^{i}, l_{a}^{i}\right)$, where in terms of the wrapping numbers $l_{a}^{i} \equiv m_{a}^{i}$ for a rectangular torus and $l_{a}^{i} \equiv 2 \tilde{m}_{a}^{i}=2 m_{a}^{i}+n_{a}^{i}$ for a tilted torus. For a stack of $N_{a}$ D6-branes along cycle $\left(n_{a}^{i}, l_{a}^{i}\right)$ we also need to include their $\Omega R$ images $N_{a^{\prime}}$ with wrapping numbers $\left(n_{a}^{i},-l_{a}^{i}\right)$. For branes on top of the O6-planes we also count branes and their images independently. So the homology three-cycles for stack $a$ 
of $N_{a}$ D6-branes and its orientifold image $a^{\prime}$ take the form

$$
\left[\Pi_{a}\right]=\prod_{i=1}^{3}\left(n_{a}^{i}\left[a_{i}\right]+2^{-\beta_{i}} l_{a}^{i}\left[b_{i}\right]\right), \quad\left[\Pi_{a^{\prime}}\right]=\prod_{i=1}^{3}\left(n_{a}^{i}\left[a_{i}\right]-2^{-\beta_{i}} l_{a}^{i}\left[b_{i}\right]\right)
$$

where $\beta_{i}=0$ if the $i$ th torus is not tilted and $\beta_{i}=1$ if it is tilted. In particular, the homology three-cycles wrapped by the four orientifold planes are (our normalization of the orientifold cycles, explained in more detail in [19], is slightly different from the one in [13]. Here we include the multiplicity of the orientifold planes which allows for a uniform treatment of rectangular and tilted tori.)

$$
\begin{array}{cl}
\Omega R: \quad\left[\Pi_{1}\right]=8\left[a_{1}\right] \times\left[a_{2}\right] \times\left[a_{3}\right], \quad \Omega R \omega:\left[\Pi_{2}\right]=-2^{3-\beta_{2}-\beta_{3}}\left[a_{1}\right] \times\left[b_{2}\right] \times\left[b_{3}\right] \\
\Omega R \theta \omega:\left[\Pi_{3}\right]=-2^{3-\beta_{1}-\beta_{3}}\left[b_{1}\right] \times\left[a_{2}\right] \times\left[b_{3}\right], \quad \Omega R \theta:\left[\Pi_{4}\right]=-2^{3-\beta_{1}-\beta_{2}}\left[b_{1}\right] \times\left[b_{2}\right] \times\left[a_{3}\right]
\end{array}
$$

If we define $\left[\Pi_{O 6}\right]=\left[\Pi_{1}\right]+\left[\Pi_{2}\right]+\left[\Pi_{3}\right]+\left[\Pi_{4}\right]$, the chiral spectrum in the open string sector can be described in terms of the intersection numbers

$$
\begin{aligned}
& I_{a b}=\left[\Pi_{a}\right]\left[\Pi_{b}\right]=2^{-k} \prod_{i=1}^{3}\left(n_{a}^{i} l_{b}^{i}-n_{b}^{i} l_{a}^{i}\right), I_{a b^{\prime}}=\left[\Pi_{a}\right]\left[\Pi_{b^{\prime}}\right]=-2^{-k} \prod_{i=1}^{3}\left(n_{a}^{i} l_{b}^{i}+n_{b}^{i} l_{a}^{i}\right) \\
& I_{a a^{\prime}}=\left[\Pi_{a}\right]\left[\Pi_{a^{\prime}}\right]=-2^{3-k} \prod_{i=1}^{3}\left(n_{a}^{i} l_{a}^{i}\right) \\
& I_{a O 6}=\left[\Pi_{a}\right]\left[\Pi_{O 6}\right]=2^{3-k}\left(-l_{a}^{1} l_{a}^{2} l_{a}^{3}+l_{a}^{1} n_{a}^{2} n_{a}^{3}+n_{a}^{1} l_{a}^{2} n_{a}^{3}+n_{a}^{1} n_{a}^{2} l_{a}^{3}\right)
\end{aligned}
$$

where $k=\beta_{1}+\beta_{2}+\beta_{3}$ is the total number of tilted tori.

The model is constrained by the tadpole cancellation conditions

$$
\sum_{a} N_{a}\left[\Pi_{a}\right]+\sum_{a} N_{a}\left[\Pi_{a^{\prime}}\right]-4\left[\Pi_{O 6}\right]=0
$$

as well as the conditions to preserve $\mathcal{N}=1$ supersymmetry in $D=4$ [6], namely each stack of $D 6$-branes should be related to the $\Omega R$-plane by an $S U(3)$ rotation. If $\theta_{i}$ is the angle the $D 6$-brane makes with the $\Omega R$-plane in the $i$ th torus, then supersymmetry requires $\theta_{1}+\theta_{2}+\theta_{3}=0 \bmod 2 \pi$. In [19] it was shown that this condition can be rewritten as

$$
\begin{array}{r}
-x_{A} l_{a}^{1} l_{a}^{2} l_{a}^{3}+x_{B} l_{a}^{1} n_{a}^{2} n_{a}^{3}+x_{C} n_{a}^{1} l_{a}^{2} n_{a}^{3}+x_{D} n_{a}^{1} n_{a}^{2} l_{a}^{3}=0 \\
-n_{a}^{1} n_{a}^{2} n_{a}^{3} / x_{A}+n_{a}^{1} l_{a}^{2} l_{a}^{3} / x_{B}+l_{a}^{1} n_{a}^{2} l_{a}^{3} / x_{C}+l_{a}^{1} l_{a}^{2} n_{a}^{3} / x_{D}<0
\end{array}
$$

where $x_{A}=\lambda, x_{B}=\lambda 2^{\beta_{2}+\beta 3} / \chi_{2} \chi_{3}, x_{C}=\lambda 2^{\beta_{1}+\beta 3} / \chi_{1} \chi_{3}, x_{D}=\lambda 2^{\beta_{1}+\beta 2} / \chi_{1} \chi_{2}$ and $\chi_{i}=$ $\left(R_{2} / R_{1}\right)_{i}$ are the complex structure moduli. $\lambda$ is a positive parameter without physical significance. 
The open string spectrum of these constructions for branes at generic angles was discussed in detail in [13]. We summarize the results in table I. In particular, the aa sector arising from open strings stretching within a single stack of $\mathrm{D} 6_{a}$-branes contains $U\left(N_{a} / 2\right)$ gauge fields as well as three adjoint $N=1$ chiral multiplets which are the moduli associated with the non-rigidness of the three-cycles the D6-branes wrap. The $a b+b a$ sector contains $I_{a b}$ chiral multiplets in the $\left(\square_{a}, \bar{\square}_{b}\right)$ representation of $U\left(N_{a} / 2\right) \times U\left(N_{b} / 2\right)$ while $a b^{\prime}+b^{\prime} a$ contains $I_{a b^{\prime}}$ chiral multiplets in the bifundamental $\left(\square_{a}, \square_{b}\right)$. Finally, the $a a^{\prime}+a^{\prime} a$ sector contains symmetric and antisymmetric representations of the $U\left(N_{a} / 2\right)$ gauge group with multiplicities given in table I. Notice that because of the change of notation from [12, 13], the multiplicities of $\square$ and $\boxminus$ have slightly different expressions here.

\begin{tabular}{|c|c|}
\hline Sector & Representation \\
\hline \hline$a a$ & $U\left(N_{a} / 2\right)$ vector multiplet \\
& 3 Adj. chiral multiplets \\
\hline$a b+b a$ & $I_{a b}\left(\square_{a}, \square_{b}\right)$ fermions \\
\hline$a b^{\prime}+b^{\prime} a$ & $I_{a b^{\prime}}\left(\square_{a}, \square_{b}\right)$ fermions \\
\hline$a a^{\prime}+a^{\prime} a$ & $-\frac{1}{2}\left(I_{a a^{\prime}}-\frac{1}{2} I_{a, O 6}\right) \square$ fermions \\
& $-\frac{1}{2}\left(I_{a a^{\prime}}+\frac{1}{2} I_{a, O 6}\right) \boxminus$ fermions \\
\hline
\end{tabular}

TABLE I: General spectrum on D6-branes at generic angles, i.e., angles that are not parallel to any O6-plane in all three tori. The spectrum is valid for both tilted and untilted tori. The models may contain additional non-chiral pieces in the $a a^{\prime}$ sector and in $a b, a b^{\prime}$ sectors with zero intersection, if the relevant branes overlap. In supersymmetric situations, scalars combine with the fermions given above to form chiral supermultiplets.

\section{FEATURES OF THE SUPERSYMMETRIC STANDARD-LIKE MODELS}

In trying to solve the tadpole and supersymmetry conditions, along with other phenomenological constraints such as three families of quarks and leptons and semi realistic gauge groups, it is very useful to classify all possible supersymmetric brane configurations that can be used in any model based on this construction. This is indeed possible because supersymmetry, in contrast to the tadpole cancellation conditions, constrains each stack of branes individually. In [19] we have classified all supersymmetric brane configurations in the context of the $\mathbf{T}^{\mathbf{6}} /\left(\mathbf{Z}_{\mathbf{2}} \times \mathbf{Z}_{\mathbf{2}}\right)$ orientifold described above. We found that a brane wrapping a 
3 -cycle which has three wrapping numbers $n_{i}, l_{i}$ equal to zero is necessarily parallel to one of the four orientifold planes. Such brane configurations (filler branes) trivially satisfy the supersymmetry conditions and so can help solve the tadpole conditions. There can be no supersymmetric brane configuration along a cycle with two vanishing wrapping numbers and hence the only other possibilities are one or no zero wrapping numbers. Each of these gives a supersymmetric configuration together with a constraint for the complex structure moduli [19]. Since there are three independent moduli parameters we can generically include only up to three such brane configurations. Otherwise the system is overconstrained. Having these building blocks at our disposal we can start looking for models with the desired gauge group and number of families.

We first consider three-family models with gauge group $U(4) \times U(2) \times U(1)$. Here $S U(4)$ subgroup of $U(4)$ corresponds to the Pati-Salam symmetry, $S U(2)$ subgroup of $U(2)$ corresponds to the $S U(2)_{L}$ of the Standard-model symmetry and $U(1)$ is the additional nonanomalous gauge symmetry associated with the Cartan generator of the $S U(2)_{R}$ symmetry of the left-right symmetric model. Namely, the obtained the Standard Model gauge group structure is a descendant of the left-right symmetric gauge group. Starting with a stack of 8 branes not parallel to the orientifold planes (these may be split into two parallel but not overlapping stacks of 6 and 2 branes giving $U(3)$ and $U(1)$ gauge groups respectively) a second stack of 4 branes is introduced to generate an additional $U(2)$ (Note that the $S U(3)$ subgroup of $U(3)$ is associated with $S U(3)_{\text {color }}$ of the Standard-model). The number of families of quarks and leptons is given by the intersections of the $U(3)$ stack with the $U(2)$ stack and its $\Omega R$-image. As mentioned in [13] the requirement of three families forces at least one of the tori to be tilted. Since all left handed fermions should transform under the same $U(3)$ representation the intersections of the $U(3)$ stack with the $U(2)$ stack and its image (in this order!) must have the same sign.

Apparently the tadpole and supersymmetry conditions for this type of models are less constraining than for the three-family $S U(5)$ GUT models in [19]. Although this makes it easier to find consistent Standard-like models it seems more difficult to find all possible models and show there are no more models of a certain type as was done for GUT models. So, instead, in table II we present some examples and compare them with the three-family model of [13]. In fact, model I.3 is precisely this earlier model but with slightly different conventions (the wrapping numbers have been assigned differently to the three two-tori). All four models share a similar gauge group and spectrum. The chiral spectra in the open string sector are tabulated in Tables IV, V, VI.

To generate right-handed quarks and leptons (and of course the appropriate assignments for the $U(1)$ hypercharge) we break the $U S p(8)$ or $U S p(10)$ factor to a number of $U(1) \mathrm{s}$ by moving the branes away from the orientifold planes. From the action of the orientifold 


\begin{tabular}{|c|c|c|c|c|c|c|c|c|c|}
\hline model I.1 & \multicolumn{9}{|c|}{$U(3) \times U(1) \times U(2) \times U S p(8) \times U S p(2) \times U S p(4)$} \\
\hline stack & $N$ & $\left(n^{1}, l^{1}\right) \times\left(n^{2}, l^{2}\right) \times\left(n^{3}, l^{3}\right)$ & $n_{\square}^{n}$ & $n_{\text {口 }}$ & $b$ & $b^{\prime}$ & 1 & 2 & 3 \\
\hline$a$ & $6+2$ & $(0,1) \times(1,-1) \times(1,-1)$ & 0 & 0 & 0 & 3 & -1 & 1 & 0 \\
\hline$b$ & 4 & $(3,-2) \times(0,1) \times(1,-1)$ & 1 & -1 & - & - & -2 & 0 & 3 \\
\hline 1 & 8 & $(1,0) \times(1,0) \times(2,0)$ & \multirow{3}{*}{\multicolumn{7}{|c|}{$2 x_{A}=2 x_{B}=3 x_{C}$}} \\
\hline 2 & 2 & $(1,0) \times(0,1) \times(0,-2)$ & & & & & & & \\
\hline 3 & 4 & $(0,1) \times(1,0) \times(0,-2)$ & & & & & & & \\
\hline model I.2 & \multicolumn{9}{|c|}{$U(3) \times U(1) \times U(2) \times U S p(8) \times U S p(2) \times U S p(4)$} \\
\hline stack & $N$ & $\left(n^{1}, l^{1}\right) \times\left(n^{2}, l^{2}\right) \times\left(n^{3}, l^{3}\right)$ & $n_{\square}$ & ${ }^{n}$ 日 & $b$ & $b^{\prime}$ & 1 & 2 & 3 \\
\hline$a$ & $6+2$ & $(0,1) \times(1,-1) \times(1,-1)$ & 0 & 0 & 1 & 2 & -1 & 1 & 0 \\
\hline$b$ & 4 & $(-1,2) \times(0,1) \times(-1,3)$ & -5 & 5 & - & - & -6 & 0 & 1 \\
\hline 1 & 8 & $(1,0) \times(1,0) \times(2,0)$ & \multirow{3}{*}{\multicolumn{7}{|c|}{$6 x_{A}=6 x_{B}=x_{C}$}} \\
\hline 2 & 2 & $(1,0) \times(0,1) \times(0,-2)$ & & & & & & & \\
\hline 3 & 4 & $(0,1) \times(1,0) \times(0,-2)$ & & & & & & & \\
\hline
\end{tabular}

TABLE II: D6-brane configurations and intersection numbers for the three-family Standard-like models (continued in table III). Here $a, b$ denote the stacks of D-branes not parallel to the orientifold planes, giving $U\left(N_{a} / 2\right)$ gauge group, while $a^{\prime}, b^{\prime}$ denote their $\Omega R$ image. 1,2,3,4 denote filler branes respectively along the $\Omega R, \Omega R \omega, \Omega R \theta \omega$ and $\Omega R \theta$ orientifold plane, resulting in a $U S p\left(N^{(i)}\right)$ gauge group. $N$ is the number of branes in each stack. The third column shows the wrapping number of the various branes. In all models only the third torus has a non-trivial complex structure. The intersection numbers between the various stacks are given in the remaining columns to the right. For example, the intersection number $I_{a c}$ between stacks $a$ and $b$ is found in row $a$ column $b$. For convenience we also list the relation among the moduli imposed by the supersymmetry conditions, as well as the gauge group for each model.

group 1 and 2 we see that a D6-brane which is away from the orientifold plane in one of the three 2-tori has one image under the orientifold group. Similarly, a brane away from the orientifold plane in two or three tori has two and three images respectively. This means that we can break $U S p(2)$ to a single $U(1)$ by moving the 3-cycle away from the orientifold plane in one torus (the original two branes on top of the orientifold plane account for a single brane away from the orientifold plane and its image). USp(4) can be broken to either two $U(1)$ s through two $U S p(2)$ s or down to a single $U(1)$ by moving the brane away from the orientifold plane in all three tori (thus generating four images). For $U S p(6)$ we can have the 


\begin{tabular}{|c|c|c|c|c|c|c|c|c|c|c|}
\hline model I.3 & \multicolumn{10}{|c|}{$U(3) \times U(1) \times U(2) \times U S p(8) \times U S p(2) \times U S p(4) \times U S p(2)$} \\
\hline stack & $N$ & $\left(n^{1}, l^{1}\right) \times\left(n^{2}, l^{2}\right) \times\left(n^{3}, l^{3}\right)$ & $n_{\square}$ & $n_{\text {足 }}$ & $b$ & $b^{\prime}$ & 1 & 2 & 3 & 4 \\
\hline$a$ & $6+2$ & $(0,1) \times(1,-1) \times(1,-1)$ & 0 & 0 & 1 & 2 & -1 & 1 & 0 & 0 \\
\hline$b$ & 4 & $(-1,1) \times(0,1) \times(-1,3)$ & -2 & 2 & - & - & -3 & 0 & 1 & 0 \\
\hline 1 & 8 & $(1,0) \times(1,0) \times(2,0)$ & \multirow{4}{*}{\multicolumn{8}{|c|}{$3 x_{A}=3 x_{B}=x_{C}$}} \\
\hline 2 & 2 & $(1,0) \times(0,1) \times(0,-2)$ & & & & & & & & \\
\hline 3 & 4 & $(0,1) \times(1,0) \times(0,-2)$ & & & & & & & & \\
\hline 4 & 2 & $(0,1) \times(0,-1) \times(2,0)$ & & & & & & & & \\
\hline model I.4 & \multicolumn{10}{|c|}{$U(3) \times U(1) \times U(2) \times U S p(10) \times U S p(4) \times U S p(2)$} \\
\hline stack & $N$ & $\left(n^{1}, l^{1}\right) \times\left(n^{2}, l^{2}\right) \times\left(n^{3}, l^{3}\right)$ & $n_{\square}$ & $n_{\text {口 }}$ & $b$ & $b$ & & 1 & 2 & 3 \\
\hline$a$ & $6+2$ & $(0,1) \times(1,-1) \times(1,-1)$ & 0 & 0 & 0 & & & -1 & 1 & 0 \\
\hline$b$ & 4 & $(1,-1) \times(-1,2) \times(1,-1)$ & -2 & -6 & - & & & -2 & 1 & 2 \\
\hline 1 & 10 & $(1,0) \times(1,0) \times(2,0)$ & \multirow{3}{*}{\multicolumn{8}{|c|}{$x_{A}=x_{B}>2 x_{C}$}} \\
\hline 2 & 4 & $(1,0) \times(0,1) \times(0,-2)$ & & & & & & & & \\
\hline 3 & 2 & $(0,1) \times(1,0) \times(0,-2)$ & & & & & & & & \\
\hline
\end{tabular}

TABLE III: D6-brane configurations and intersection numbers for the three-family Standard-like models. Continued from table II.

following breaking patterns

$$
\begin{array}{r}
U S p(6) \rightarrow U S p(2) \times U S p(2) \times U S p(2) \rightarrow U(1) \times U(1) \times U(1) \\
U S p(6) \rightarrow U S p(4) \times U S p(2) \rightarrow \ldots \\
U S p(6) \rightarrow U(1) \times U(1)
\end{array}
$$

where the last scheme arises when two of the original six branes are displaced away from the orientifold plane in two tori, thus each gaining two images under the orientifold group. Since the $U(1)$ s obtained this way are non-anomalous they do not acquire string scale mass and so extend the gauge group beyond the Standard Model. To avoid having too many such factors surviving we break $U S p(8)$ and $U S p(10)$ as

$$
\begin{array}{r}
U S p(8) \rightarrow U S p(4) \times U S p(4) \rightarrow U(1) \times U(1) \\
U S p(10) \rightarrow U S p(8) \times U S p(2) \rightarrow U(1) \times U(1) \times U(1)
\end{array}
$$

resulting in the minimal number of $U(1) \mathrm{s}$.

If we denote by $Q_{1}, Q_{2}$ and $Q_{3}$ the $U(1)$ factor of the corresponding $U(n)$, the linear combination $Q_{3} / 3-Q_{1}=B-L$ is non-anomalous because it corresponds to a generator in 


\begin{tabular}{|c||c||c|c|c|c|c||c|c|c|}
\hline I.1 & $S U(3) \times S U(2) \times U S p(2) \times U S p(4)$ & $Q_{3}$ & $Q_{1}$ & $Q_{2}$ & $Q_{8}$ & $Q_{8}^{\prime}$ & $Q_{Y}$ & $Q_{8}-Q_{8}^{\prime}$ & Field \\
\hline \hline$a b^{\prime}$ & $3 \times(3,2,1,1)$ & 1 & 0 & 1 & 0 & 0 & $\frac{1}{6}$ & 0 & $Q_{L}$ \\
& $3 \times(1,2,1,1)$ & 0 & 1 & 1 & 0 & 0 & $-\frac{1}{2}$ & 0 & $L$ \\
& $2 \times(\overline{3}, 1,1,1)$ & -1 & 0 & 0 & \pm 1 & 0 & $\frac{1}{3},-\frac{2}{3}$ & \pm 1 & $\bar{U}, \bar{D}$ \\
& $2 \times(\overline{3}, 1,1,1)$ & -1 & 0 & 0 & 0 & \pm 1 & $\frac{1}{3},-\frac{2}{3}$ & $\mp 1$ & $\bar{U}, \bar{D}$ \\
& $2 \times(1,1,1,1)$ & 0 & -1 & 0 & \pm 1 & 0 & 1,0 & \pm 1 & $\bar{E}, \bar{N}$ \\
& $2 \times(1,1,1,1)$ & 0 & -1 & 0 & 0 & \pm 1 & 1,0 & $\mp 1$ & $\bar{E}, \bar{N}$ \\
& $(3,1,2,1)$ & 1 & 0 & 0 & 0 & 0 & $\frac{1}{6}$ & 0 & \\
& $(1,1,2,1)$ & 0 & 1 & 0 & 0 & 0 & $\frac{1}{6}$ & 0 & \\
$b 1$ & $2 \times 2 \times(1, \overline{2}, 1,1)$ & 0 & 0 & -1 & \pm 1 & 0 & $\pm \frac{1}{2}$ & \pm 1 & $H_{U}, H_{D}$ \\
& $2 \times 2 \times(1, \overline{2}, 1,1)$ & 0 & 0 & -1 & 0 & \pm 1 & $\pm \frac{1}{2}$ & $\mp 1$ & $H_{U}, H_{D}$ \\
$b 3$ & $(1,3,1,1)$ & 0 & 0 & 1 & 0 & 0 & 0 & 0 & \\
$b \square$ & $(1,1,1,1)$ & 0 & 0 & 2 & 0 & 0 & 0 & 0 & \\
$b_{\square}$ & $3 \times(1,2,1,4)$ & 0 & 0 & -2 & 0 & 0 & 0 & 0 & \\
\hline
\end{tabular}

TABLE IV: The chiral spectrum in the open string sector of model I.1. All three families of lefthanded quarks and leptons appear at the same intersection. There are eight Higgs doublets of each type in this model.

$S U$ (4) when all eight branes are coincident. Here $B=Q_{3}$ is the baryon number and $L=Q_{1}$ plays the role of lepton number. In models I.1, I.2 and I.3 there are three non anomalous $U(1)$ factors, namely $B-L, Q_{8}$ and $Q_{8}^{\prime}$, the last two coming from breaking $U S p(8)$, and two anomalous, $B+L$ and $Q_{2}$. In model I.4 there is an extra non-anomalous $U(1), Q_{10}$, coming from the $U S p(2)$ of $U S p(10)$. The hypercharge is defined as the non-anomalous linear combination

$$
Q_{Y}=\frac{1}{6} Q_{3}-\frac{1}{2} Q_{1}+\frac{1}{2}\left(Q_{8}+Q_{8}^{\prime}+Q_{10}\right) .
$$

The phenomenology and the tree-level gauge couplings of the three-family model in [13] were discussed in detail in [22]. Yukawa couplings in this context were discussed in [23] and more recently studied in detail in $[27,28,29]$. The models we present here have very similar properties and so we will simply point out the essential differences.

A common feature of the new models is that they have less filler branes than the original model I.3. In fact model I.4 is the first example that includes a stack with no zero angles relative to the $\Omega R$ plane, and hence there is an additional complex structure modulus, that is not fixed. In fact there is a two-parameter family of such models in moduli space. Models I.1 and I.4 give three families of left-handed quarks and leptons from the same intersection, in 


\begin{tabular}{|c|c|c|c|c|c|c|c|c|c|}
\hline I.2 & $S U(3) \times S U(2) \times U S p(2) \times U S p(4)$ & $Q_{3}$ & $Q_{1}$ & $Q_{2}$ & $Q_{8}$ & $Q_{8}^{\prime}$ & $Q_{Y}$ & $Q_{8}-Q_{8}^{\prime}$ & Field \\
\hline \multirow[t]{2}{*}{$a b$} & $(3, \overline{2}, 1,1)$ & 1 & 0 & -1 & 0 & 0 & $\frac{1}{6}$ & 0 & $Q_{L}$ \\
\hline & $(1, \overline{2}, 1,1)$ & 0 & 1 & -1 & 0 & 0 & $-\frac{1}{2}$ & 0 & $L$ \\
\hline \multirow[t]{2}{*}{$a b^{\prime}$} & $2 \times(3,2,1,1)$ & 1 & 0 & 1 & 0 & 0 & $\frac{1}{6}$ & 0 & $Q_{L}$ \\
\hline & $2 \times(1,2,1,1)$ & 0 & 1 & 1 & 0 & 0 & $-\frac{1}{2}$ & 0 & $L$ \\
\hline \multirow[t]{4}{*}{$a 1$} & $2 \times(\overline{3}, 1,1,1)$ & -1 & 0 & 0 & \pm 1 & 0 & $\frac{1}{3},-\frac{2}{3}$ & \pm 1 & $\bar{U}, \bar{D}$ \\
\hline & $2 \times(\overline{3}, 1,1,1)$ & -1 & 0 & 0 & 0 & \pm 1 & $\frac{1}{3},-\frac{2}{3}$ & $\mp 1$ & $\bar{U}, \bar{D}$ \\
\hline & $2 \times(1,1,1,1)$ & 0 & -1 & 0 & \pm 1 & 0 & 1,0 & \pm 1 & $\bar{E}, \bar{N}$ \\
\hline & $2 \times(1,1,1,1)$ & 0 & -1 & 0 & 0 & \pm 1 & 1,0 & $\mp 1$ & $\bar{E}, \bar{N}$ \\
\hline \multirow[t]{2}{*}{$a 2$} & $(3,1,2,1)$ & 1 & 0 & 0 & 0 & 0 & $\frac{1}{6}$ & 0 & \\
\hline & $(1,1,2,1)$ & 0 & 1 & 0 & 0 & 0 & $\frac{1}{6}$ & 0 & \\
\hline \multirow[t]{2}{*}{$b 1$} & $2 \times 6 \times(1, \overline{2}, 1,1)$ & 0 & 0 & -1 & \pm 1 & 0 & $\pm \frac{1}{2}$ & \pm 1 & $H_{U}, H_{D}$ \\
\hline & $2 \times 6 \times(1, \overline{2}, 1,1)$ & 0 & 0 & -1 & 0 & \pm 1 & $\pm \frac{1}{2}$ & $\mp 1$ & $H_{U}, H_{D}$ \\
\hline$b 3$ & $(1,2,1,4)$ & 0 & 0 & 1 & 0 & 0 & 0 & 0 & \\
\hline$b_{\square}$ & $5 \times(1, \overline{3}, 1,1)$ & 0 & 0 & -2 & 0 & 0 & 0 & 0 & \\
\hline${ }^{b}$ & $5 \times(1,1,1,1)$ & 0 & 0 & 2 & 0 & 0 & 0 & 0 & \\
\hline
\end{tabular}

TABLE V: The chiral spectrum of model I.2. The model contains 24 Higgs doublets of each type.

contrast to the rest of the models where two families are obtained from one intersection and the third from another. The original model had 24 Higgs doublets $\left(12 H_{U}\right.$ and $\left.12 H_{D}\right)$. This is somewhat improved in model I.1 ( $8+8$ Higgs doublets) and I.4 (10+10 Higgs doublets) although model I.2 contains 24+24 Higgs doublets. Additionally, model I.4 has five families of right handed quarks and leptons compared to four in the rest of the models. In fact, since the right handed quarks and leptons are obtained by breaking $U S p(8)$ to two $U(1) \mathrm{s}$, one can get less families by less breaking of $U S p(8)$ but then the spectrum would contain more exotic multiplets. The existence of extra non-anomalous $U(1) \mathrm{s}$ is a generic feature of these supersymmetric models. Typically, one of these additional $U(1)$ 's can be broken by the scalar component of $\bar{N}$, i.e. the right-handed neutrino super-multiplet. However, for the additional $U(1)$ s there are no Higgs field candidates to spontaneously break them (see [22] for a discussion). 


\begin{tabular}{|c|c|c|c|c|c|c|c|c|c|}
\hline I.3 & $S U(3) \times S U(2) \times U S p(2)_{2} \times U S p(4) \times U S p(2)_{4}$ & $Q_{3}$ & $Q_{1}$ & $Q_{2}$ & $Q_{8}$ & $Q_{8}^{\prime}$ & $Q_{Y}$ & $Q_{8}-Q_{8}^{\prime}$ & Field \\
\hline \multirow[t]{2}{*}{$a b$} & $(3, \overline{2}, 1,1,1)$ & 1 & 0 & -1 & 0 & 0 & $\frac{1}{6}$ & 0 & $Q_{L}$ \\
\hline & $(1, \overline{2}, 1,1,1)$ & 0 & 1 & -1 & 0 & 0 & $-\frac{1}{2}$ & 0 & $L$ \\
\hline \multirow[t]{2}{*}{$a b^{\prime}$} & $2 \times(3,2,1,1)$ & 1 & 0 & 1 & 0 & 0 & $\frac{1}{6}$ & 0 & $Q_{L}$ \\
\hline & $2 \times(1,2,1,1,1)$ & 0 & 1 & 1 & 0 & 0 & $-\frac{1}{2}$ & 0 & $L$ \\
\hline \multirow[t]{4}{*}{$a 1$} & $2 \times(\overline{3}, 1,1,1,1)$ & -1 & 0 & 0 & \pm 1 & 0 & $\frac{1}{3},-\frac{2}{3}$ & \pm 1 & $\bar{U}, \bar{D}$ \\
\hline & $2 \times(\overline{3}, 1,1,1,1)$ & -1 & 0 & 0 & 0 & \pm 1 & $\frac{1}{3},-\frac{2}{3}$ & $\mp 1$ & $\bar{U}, \bar{D}$ \\
\hline & $2 \times(1,1,1,1,1)$ & 0 & -1 & 0 & \pm 1 & 0 & 1,0 & \pm 1 & $\bar{E}, \bar{N}$ \\
\hline & $2 \times(1,1,1,1,1)$ & 0 & -1 & 0 & 0 & \pm 1 & 1,0 & $\mp 1$ & $\bar{E}, \bar{N}$ \\
\hline \multirow[t]{2}{*}{$a 2$} & $(3,1,2,1,1)$ & 1 & 0 & 0 & 0 & 0 & $\frac{1}{6}$ & 0 & \\
\hline & $(1,1,2,1,1)$ & 0 & 1 & 0 & 0 & 0 & $\frac{1}{6}$ & 0 & \\
\hline \multirow[t]{2}{*}{$b 1$} & $2 \times 3 \times(1, \overline{2}, 1,1,1)$ & 0 & 0 & -1 & \pm 1 & 0 & $\pm \frac{1}{2}$ & \pm 1 & $H_{U}, H_{D}$ \\
\hline & $2 \times 3 \times(1, \overline{2}, 1,1,1)$ & 0 & 0 & -1 & 0 & \pm 1 & $\pm \frac{1}{2}$ & $\mp 1$ & $H_{U}, H_{D}$ \\
\hline$b 3$ & $(1,2,1,4,1)$ & 0 & 0 & 1 & 0 & 0 & 0 & 0 & \\
\hline$b \square$ & $2 \times(1, \overline{3}, 1,1,1)$ & 0 & 0 & -2 & 0 & 0 & 0 & 0 & \\
\hline$b_{日}$ & $2 \times(1,1,1,1,1)$ & 0 & 0 & 2 & 0 & 0 & 0 & 0 & \\
\hline
\end{tabular}

TABLE VI: The chiral spectrum of model I.3. This is precisely the first three-family supersymmetric model obtained in $[12,13,14]$.

\section{A. A left-right symmetric model}

Here we present an example of a supersymmetric left-right symmetric model with three generations of quarks and leptons. The brane configuration is shown in Table VIII and the chiral spectrum in the open string sector is tabulated in Table IX. The electromagnetic charge is obtained as

$$
Q_{e m}=I_{3 L}+I_{3 R}+\frac{1}{2}(B-L)
$$

where $I_{3 L}, I_{3 R}$ are the Cartan generators of $S U(2)_{L}$ and $S U(2)_{R}$, respectively, and $B-L$ is identified as before with the $U(1)$ in the breaking of $S U(4) \rightarrow S U(3) \times U(1)_{B-L}$.

The Higgs sector of the model, however, contains 16 Higgs bi-doublets. In addition, the symmetric multiplets coming from $S U(2)_{L}$ and $S U(2)_{R}$ are not charged under $B-L$ and hence they cannot be used to break $S U(2)_{R}$. Nevertheless, $S U(2)_{R}$ could be broken to $U(1)$ by separating the two branes but, again, there is no mechanism to break this non-anomalous $U(1)$. 


\begin{tabular}{|c|c|c|c|c|c|c|c|c|c|c|}
\hline I.4 & $S U(3) \times S U(2) \times U S p(4) \times U S p(2)$ & $Q_{3}$ & $Q_{1}$ & $Q_{2}$ & $Q_{8}$ & $Q_{8}^{\prime}$ & $Q_{10}$ & $Q_{Y}$ & $Q_{8}-Q_{8}^{\prime}$ & Field \\
\hline \multirow[t]{2}{*}{$a b^{\prime}$} & $3 \times(3,2,1,1)$ & 1 & 0 & 1 & 0 & 0 & 0 & $\frac{1}{6}$ & 0 & $Q_{L}$ \\
\hline & $3 \times(1,2,1,1)$ & 0 & 1 & 1 & 0 & 0 & 0 & $-\frac{1}{2}$ & 0 & $L$ \\
\hline \multirow[t]{6}{*}{$a 1$} & $2 \times(\overline{3}, 1,1,1)$ & -1 & 0 & 0 & \pm 1 & 0 & 0 & $\frac{1}{3},-\frac{2}{3}$ & \pm 1 & $\bar{U}, \bar{D}$ \\
\hline & $2 \times(\overline{3}, 1,1,1)$ & -1 & 0 & 0 & 0 & \pm 1 & 0 & $\frac{1}{3},-\frac{2}{3}$ & $\mp 1$ & $\bar{U}, \bar{D}$ \\
\hline & $(\overline{3}, 1,1,1)$ & -1 & 0 & 0 & 0 & 0 & \pm 1 & $\frac{1}{3},-\frac{2}{3}$ & 0 & $\bar{U}, \bar{D}$ \\
\hline & $2 \times(1,1,1,1)$ & 0 & -1 & 0 & \pm 1 & 0 & 0 & 1,0 & \pm 1 & $\bar{E}, \bar{N}$ \\
\hline & $2 \times(1,1,1,1)$ & 0 & -1 & 0 & 0 & \pm 1 & 0 & 1,0 & $\mp 1$ & $\bar{E}, \bar{N}$ \\
\hline & $(1,1,1,1)$ & 0 & -1 & 0 & 0 & 0 & \pm 1 & 1,0 & 0 & $\bar{E}, \bar{N}$ \\
\hline \multirow[t]{2}{*}{$a 2$} & $(3,1,4,1)$ & 1 & 0 & 0 & 0 & 0 & 0 & $\frac{1}{6}$ & 0 & \\
\hline & $(1,1,4,1)$ & 0 & 1 & 0 & 0 & 0 & 0 & $\frac{1}{6}$ & 0 & \\
\hline \multirow[t]{3}{*}{$b 1$} & $2 \times 2 \times(1, \overline{2}, 1,1)$ & 0 & 0 & -1 & \pm 1 & 0 & 0 & $\pm \frac{1}{2}$ & \pm 1 & $H_{U}, H_{D}$ \\
\hline & $2 \times 2 \times(1, \overline{2}, 1,1)$ & 0 & 0 & -1 & 0 & \pm 1 & 0 & $\pm \frac{1}{2}$ & $\mp 1$ & $H_{U}, H_{D}$ \\
\hline & $2 \times(1, \overline{2}, 1,1)$ & 0 & 0 & -1 & 0 & 0 & \pm 1 & $\pm \frac{1}{2}$ & 0 & $H_{U}, H_{D}$ \\
\hline$b 3$ & $2 \times(1,2,1,2)$ & 0 & 0 & 1 & 0 & 0 & 0 & 0 & 0 & \\
\hline$b_{\square}$ & $2 \times(1, \overline{3}, 1,1)$ & 0 & 0 & -2 & 0 & 0 & 0 & 0 & 0 & \\
\hline 曰日 & $6 \times(1,1,1,1)$ & 0 & 0 & -2 & 0 & 0 & 0 & 0 & 0 & \\
\hline
\end{tabular}

TABLE VII: The chiral spectrum of model I.4. As for model I.1 all three families of left-handed quarks and leptons come from the same intersection but here there are twelve Higgs doublets of each type as in the original model I.3.

\section{CONCLUSIONS}

By generalizing the original supersymmetric constructions within $\mathbf{T}^{\mathbf{6}} /\left(\mathbf{Z}_{\mathbf{2}} \times \mathbf{Z}_{\mathbf{2}}\right)$ orientifolds with intersecting D6-branes we presented new supersymmetric Standard-like models. In particular we allowed for D6-branes wrapping most general three-cycles, which are product of one-cycles on each two-torus, and allowing for one two-torus to be tilted. As a consequence we obtained new models with the three families of quarks and leptons and the Standard-model gauge group as a part of the gauge structure. Some of the models have fewer Higgs doublets (however, still at least $8 H_{U}$ and $8 H_{D}$ Higgs doublets). We have also found models that have all the three families of left-handed quarks (and leptons) arising from the same intersection sector. This feature may allow potentially for a new pattern of Yukawa couplings of quarks and leptons to Higgs doublets. (In the original model [12, 13] one family came from a different sector and as a consequence did not have Yukawa couplings to the Higgs doublets.) 


\begin{tabular}{|c||c|c||c|c|c|c|c|c|c|c|}
\hline \multicolumn{1}{|c||}{ model II } & \multicolumn{1}{|c|}{$U(4) \times U(2)_{R} \times U(2)_{L} \times U S p(2) \times U S p(2)$} \\
\hline \hline stack & $N$ & $\left(n^{1}, l^{1}\right) \times\left(n^{2}, l^{2}\right) \times\left(n^{3}, l^{3}\right)$ & $n_{\square}$ & $n$ & $b$ & $b^{\prime}$ & $c$ & $c^{\prime}$ & 2 & 4 \\
\hline \multirow{2}{*}{$a$} & 8 & $(-1,1) \times(-1,0) \times(1,1)$ & 0 & 0 & -1 & -2 & 1 & 2 & -1 & 1 \\
$b$ & 4 & $(-1,0) \times(-2,1) \times(1,3)$ & 5 & -5 & - & - & 16 & 0 & 0 & 6 \\
$c$ & 4 & $(0,-1) \times(2,1) \times(3,1)$ & -5 & 5 & - & - & - & - & -6 & 0 \\
\hline 2 & 2 & $(1,0) \times(0,1) \times(0,-2)$ & \multicolumn{10}{|c|}{$x_{A}=x_{C}=6 x_{B}$} \\
4 & 2 & $(0,1) \times(0,-1) \times(2,0)$ & \multicolumn{10}{c|}{} \\
\hline
\end{tabular}

TABLE VIII: D6-brane configurations and intersection numbers for the three-family left-right symmetric model

\begin{tabular}{|c|c|c|c|c|c|c|c|}
\hline II & $S U(4) \times S U(2)_{R} \times S U(2)_{L} \times U S p(2) \times U S p(2)$ & $Q_{4}$ & $Q_{2}$ & $Q_{2}^{\prime}$ & $Q_{e m}$ & $B-L$ & Field \\
\hline$a b$ & $(\overline{4}, 2,1,1,1)$ & -1 & 1 & 0 & $\frac{1}{3},-\frac{2}{3}, 1,0$ & $-\frac{1}{3}, 1$ & $Q_{R}, R$ \\
\hline$a b^{\prime}$ & $2 \times(\overline{4}, \overline{2}, 1,1,1)$ & -1 & -1 & 0 & $\frac{1}{3},-\frac{2}{3}, 1,0$ & $-\frac{1}{3}, 1$ & $Q_{R}, R$ \\
\hline$a c$ & $(4,1, \overline{2}, 1,1)$ & 1 & 0 & -1 & $\frac{2}{3},-\frac{1}{3}, 0,-1$ & $\frac{1}{3},-1$ & $Q_{L}, L$ \\
\hline$a c^{\prime}$ & $2 \times(4,1,2,1,1)$ & 1 & 0 & 1 & $\frac{2}{3},-\frac{1}{3}, 0,-1$ & $\frac{1}{3},-1$ & $Q_{L}, L$ \\
\hline$a 2$ & $(\overline{4}, 1,1,2,1)$ & -1 & 0 & 0 & $-\frac{1}{6}, \frac{1}{2}$ & $-\frac{1}{3}, 1$ & \\
\hline$a 4$ & $(4,1,1,1,2)$ & 1 & 0 & 0 & $\frac{1}{6},-\frac{1}{2}$ & $\frac{1}{3},-1$ & \\
\hline$b c$ & $16 \times(1,2, \overline{2}, 1,1)$ & 0 & 1 & -1 & $0,0, \pm 1$ & 0 & $H$ \\
\hline$b 4$ & $6 \times(1,2,1,1,2)$ & 0 & 1 & 0 & $\pm \frac{1}{2}$ & 0 & \\
\hline$c 2$ & $6 \times(1,1, \overline{2}, 2,1)$ & 0 & 0 & -1 & $\pm \frac{1}{2}$ & 0 & \\
\hline$b_{\square}$ & $5 \times(1,3,1,1,1)$ & 0 & 2 & 0 & $0, \pm 1$ & 0 & \\
\hline${ }^{b} \boxminus$ & $5 \times(1,1,1,1,1)$ & 0 & -2 & 0 & 0 & 0 & \\
\hline$\square$ & $5 \times(1,1, \overline{3}, 1,1)$ & 0 & 0 & -2 & $0, \pm 1$ & 0 & \\
\hline 9 & $5 \times(1,1,1,1,1)$ & 0 & 0 & 2 & 0 & 0 & \\
\hline
\end{tabular}

TABLE IX: The chiral spectrum in the open string sector of model II

We also obtained a genuinely left-right symmetric model with three copies of left-handed and right-handed quarks and leptons. Nevertheless, this model still has a large number (16) of Higgs bi-doublets, as well as chiral matter charged under the left-right symmetric gauge group and the additional gauge sector.

In these models, the origin of the hypercharge is within left-right symmetric (Pati-Salam) gauge group structure, i.e. the non-anomalous hypercharge is obtained as a linear combination of non-anomalous U(1) charges that are a part of the Pati-Salam gauge group [30]. It 
would be interesting to generalize these constructions to cases where the hypercharge would not be a descendant of the left-right symmetric model. We hope to address these types of models in the future.

The models presented in this paper all have additional, semi-hidden, gauge group structure that is typically confining, i.e. the additional gauge group factors have negative beta functions. This sector may therefore allow for a possibility of dynamical supersymmetry breaking and moduli stabilization as addressed for the original model [12, 13] in [24]. It would be very interesting to address dynamical supersymmetry breaking for the new classes of Standard-like models, presented in this paper.

\section{Acknowledgments}

We would like to thank Paul Langacker and Gary Shiu for useful discussions. M.C. would like to thank the New Center for Theoretical Physics at Rutgers University and the Institute for Advanced Study, Princeton, for hospitality and support during the course of this work. Research supported in part by DOE grant DOE-FG02-95ER40893, NATO linkage grant No. 97061 (M.C.) and Fay R. and Eugene L. Langberg Chair (M.C.).

[1] R. Blumenhagen, L. Görlich, B. Körs and D. Lüst, JHEP 0010 (2000) 006.

[2] G. Aldazabal, S. Franco, L. E. Ibáñez, R. Rabadán and A. M. Uranga, Journal of Mathematical Physics, vol. 42, number 7, p. 3103, hep-th/0011073; JHEP 0102 (2001) 047.

[3] R. Blumenhagen, B. Körs and D. Lüst, JHEP 0102 (2001) 030.

[4] L. E. Ibáñez, F. Marchesano and R. Rabadán, hep-th/0105155.

[5] C. Angelantonj, I. Antoniadis, E. Dudas and A. Sagnotti, Phys. Lett. B 489 (2000) 223.

[6] M. Berkooz, M. R. Douglas and R. G. Leigh, Nucl. Phys. B 480 (1996) 265.

[7] C. Bachas, hep-th/9503030.

[8] S. Förste, G. Honecker and R. Schreyer, Nucl. Phys. B 593 (2001) 127; JHEP 0106 (2001) 004 .

[9] R. Blumenhagen, B. Körs and D. Lüst, T. Ott, Nucl. Phys. B616 (2001) 3.

[10] D. Bailin, G. V. Kraniotis, and A. Love, Phys. Lett. B 530, 202 (2002); Phys. Lett. B 547, 43 (2002); hep-th/0210219; hep-th/0212112.

[11] C. Kokorelis, JHEP 0209, 029 (2002); JHEP 0208, 036 (2002); hep-th/0207234; JHEP 0211, 027 (2002); hep-th/0210200.

[12] M. Cvetič, G. Shiu and A. M. Uranga, Phys. Rev. Lett. 87, 201801 (2001). 
[13] M. Cvetič, G. Shiu and A. M. Uranga, Nucl. Phys. B 615, 3 (2001).

[14] M. Cvetič , G. Shiu and A. M. Uranga, hep-th/0111179.

[15] M. Atiyah and E. Witten, hep-th/0107177.

[16] E. Witten, hep-th/0108165.

[17] B. Acharya and E. Witten, hep-th/0109152.

[18] T. Friedmann and E. Witten, hep-th/0211269.

[19] M. Cvetič, I. Papadimitriou and G. Shiu, hep-th/0212177, accepted for publication in Nucl. Phys. B.

[20] R. Blumenhagen, L. Gorlich and T. Ott, hep-th/0211059.

[21] G. Honecker, hep-th/0303015.

[22] M. Cvetič, P. Langacker and G. Shiu, Phys. Rev. D 66, 066004 (2002).

[23] M. Cvetič, P. Langacker and G. Shiu, Nucl. Phys. B 642, 139 (2002).

[24] M. Cvetič, P. Langacker and J. Wang, "Dynamical Supersymmetry Breaking and Standardlime Models with Intersecting D6-branes", RUNHETC-2002-47, to appear.

[25] D. Cremades, L. E. Ibanez and F. Marchesano, hep-ph/0212064.

[26] D. Lüst and S. Stieberger, hep-th/0302221.

[27] D. Cremades, L. E. Ibanez and F. Marchesano, hep-th/0302105.

[28] M. Cvetič and I. Papadimitriou, hep-th/0303083.

[29] S. A. Abel and A. W. Owen, hep-th/0303124.

[30] J. C. Pati and A. Salam, Phys. Rev. D 8 (1973) 1240. 